\title{
Characterization of Lipid Productivity and Fatty Acid Profile of Three Fast-Growing Microalgae Isolated from Bengkulu for Possible Use in Health Application
}

Swastika Praharyawan*, Delicia Yunita Rahman, Dwi Susilaningsih

Research Center for Biotechnology, Indonesian Institute of Sciences (LIPI), Bogor Indonesia

\section{ABSTRACT}

Three strains of fast-growing microalgae were investigated in this study for their potential of lipid production and its possible use in health application. Lipid content, lipid productivity, and fatty acids profile of the 3 microalgae strains were also analyzed. The strain of LBB13-2-AL045 and LBB13-2-AL048 possessed highest lipid content $(49.08 \pm 0.25 \%)$ and lipid productivity $\left(40.27 \pm 1.91 \mathrm{mg} . \mathrm{L}^{-1}\right.$. day $\left.^{-1}\right)$, respectively, among the other tested strains. The fatty acids profile from the 3 strains exhibited its possible use in health application. The two strains of LBB13-2AL046 and LBB13-2-AL048 possessed high valuable fatty acids of polyunsaturated fatty acids (PUFA) omega-3 and omega-6, whereas LBB13-2- AL045 possessed balance ratio of saturated fatty acid (SFA) : monounsaturated fatty acid (MUFA) : PUFA $(1: 1.3: 1)$ as is recommended by American Heart Association (AHA) $(1: 1.4: 0.8)$. The high content of omega- 3 and omega- 6 fatty acids indicated that the algal lipids of two strains (LBB13-2-AL046 and LBB13-2-AL048) were potential to be applied in cardiovascular health. The balance ratio of SFA : MUFA: PUFA as is recommended by AHA indicated that the algal oil of LBB13-2-AL045 strain was recommended in order to generate the best LDL/HDL ratio.

Keywords: Fatty acid, health, lipid, microalgae, monounsaturated fatty acid (MUFA), polyunsaturated fatty acids (PUFA), saturated fatty acid (SFA)

\section{INTRODUCTION}

Microalgae are photosynthetic microorganisms which are easy to cultivate. Microalgae receive considerable attraction as alternative renewable and natural resources because of their fast and easy grow and the fact they only need water, sunlight, and carbon dioxide for cultivation [1]. Microalgae are suitable for many fields of application, for instance animal feedstock, human nutrition, medical treatments and therapeutical, and biodiesel feedstock alternative [2,3]. As some microalgae have different composition of carbohydrates, proteins, and lipids, furthermore it is necessary to screen suitable microalgae for certain product.

Traditionally, microalgae have an enormous valuable nutrient sources for health supplements. Microalgae are known to have quite abundant of long-chain polyunsaturated fatty acid (LC-PUFA) such as omega-3 and omega-6 fatty acids [4,5]. Previous study from ${ }^{*}$ Corresponding author:

Swastika Praharyawan

Research Center for Biotechnology

Indonesian Institute of Sciences (LIPI)

Jalan Raya Bogor Km 46, Bogor 16911, Indonesia

E-mail: swastika.praharyawan@gmail.com
Chen et al. [6] showed that Nitzchialaevis is potentially able to produce eicosapentaenoic acid (EPA, 20:5,n-3). In microalgae, LC-PUFAs are predominantly located in the polar membrane lipids [7]. Omega-3 and omega-6 are essential fatty acids for humans as well as for animal, but it can't be synthesized by themselves [8]. Therefore they need a fatty acids supply from outside, for example from fish oil or vegetables [9]. These fatty acids are essential for regulation of some biological function and prevention of arrhythmia, atherosclerosis, cardiovascular disease and cancer [10,11].

Recently, commercial LC-PUFAs are produced from fish, such as salmon, mullet and mackerel [5]. One disadvantage of this commercial produced LCPUFAs in comparison to the microalgae's one is that it is unhealthy due to the fact, that these fishes are contaminated with environmental pollutants (e.g., mercury and dioxin), which are accumulated in the marine food

\section{How to cite:}

Praharyawan S, Rahman DY, Susilaningsih D (2016) Characterization of lipid productivity and fatty acid profile of three fast-growing microalgae isolated from Bengkulu for possible use in health application. J. Trop. Life. Science 6 (2): $79-85$. 
chain and can be released in the human metabolism [12]. In marine aquaculture, LC-PUFAs are primarily formed by phytoplankton, in this case, microalgae, and transferred to herbivorous zooplankton [13]. Therefore, microalgae are some of the most important microorganisms as feed stock in aquaculture, due to their nutrition value and ability to synthesis LC-PUFA. Moreover, the production system of microalgae is relatively easy to be controlled, whether for its technical or biological parameters.

As widely accepted, Indonesia is known for its high diversity of microorganisms, including microalgae. We are focusing study on collecting and screening the specific function of the Indonesian microalgae. In this regard, three strains of selected microalgae from Bengkulu province, Indonesia, have been evaluated. The objectives of the present study were: (1) investigated biomass and lipid productivity of the three microalgae strains, and (2) analyzed the fatty acids profile of the microalgae strains. In this paper, we discuss the potential use of microalgae oil in health application based on its fatty acids profile.

\section{MATERIALS AND METHODS}

\section{Microalgae culture conditions}

The strain was obtained from the culture collection of the Research Center for Biotechnology, Indonesia Institute of Sciences, Indonesia. The strain was isolated from freshwater Lake in Bengkulu province, Indonesia, the strains were LBB13-2-AL045, LBB13-2-AL046, and LBB13-2-AL048. The culture was maintained and grown in a medium containing $(\mathrm{mM}) 1.65 \mathrm{NaNO}_{3}$; $0.275 \mathrm{NH}_{4} \mathrm{NO}_{3} ; 0.12 \quad \mathrm{MgSO}_{4} .7 \mathrm{H}_{2} \mathrm{O} ; 0.074 \quad \mathrm{KH}_{2} \mathrm{PO}_{4}$; $0.029 \mathrm{~K}_{2} \mathrm{HPO}_{4} ; 0.068 \mathrm{CaCl}_{2} .2 \mathrm{H}_{2} \mathrm{O} ; 0.1 \mathrm{CaCO}_{3} ; 0.008$ $\mathrm{C}_{6} \mathrm{H}_{5} \mathrm{FeO}_{7}$; and $0.01 \mathrm{C}_{6} \mathrm{H}_{8} \mathrm{O}_{7}$. Cells were grown in a $600 \mathrm{~mL}$ bottle containing $500 \mathrm{~mL}$ of the medium with aeration and under continuous illumination. The dry biomass and total lipid content were measured when the strains had reached a late stationer stage in their growth.

\section{Microalgal growth properties}

The three selected strains of freshwater microalgae were inoculated to $500 \mathrm{~mL}$ aerated bottle and cultured at room temperature under continuous illumination at approximately $40 \mu \mathrm{mol}$ photons $\mathrm{m}^{-2} \cdot \mathrm{s}^{-1}$ for 20 days. The specific growth rate of each strain was calculated based on the equation:

$$
\mu=\ln \left(N_{y} / N x\right) /(t y-t x)
$$

Ny : dry weight of the biomass at the start (tx)

$\mathrm{Nx} \quad$ : dry weight of the biomass at the end (ty)

Biomass productivity (Pdwt) was determined as the dry biomass produced during the logarithmic growth phase. While lipid productivity (Lp) was calculated according to the equation [14]:

$$
\mathrm{Lp}=\mathrm{Pdwt} \times \mathrm{Lc}
$$

$$
\begin{array}{ll}
\text { Lp } & \text { : Lipid productivity } \\
\text { Pdwt } & : \text { Biomass productivity } \\
\text { Lc } & : \text { Lipid content }\left(\mathrm{mg} \cdot \mathrm{L}^{-1} / \text { day }\right)
\end{array}
$$

\section{Determination of lipid content}

Lipid content reported as percentage of the total biomass (in \% dry weight). Lipid extraction was conducted by adapting the modified method from Ryckebosch et al [15]. Chloroform : methanol $=1: 1$ were used as solvent in the microalgal lipid extraction. $6 \mathrm{~mL}$ of solvent was added to $100 \mathrm{mg}$ microalgae biomass and the tube was vortex mixed for $30 \mathrm{~s} .2 \mathrm{~mL}$ of solvent and water were then added and the tube was vortex mixed again and subsequently centrifuged at 2000 $\mathrm{rpm}$ for $10 \mathrm{~min}$. The aqueous layer was removed and the solvent layer was transferred into the clear tube. The remaining solid were re-extracted with $4 \mathrm{~mL}$ solvent. The re-extraction of lipid was repeated until the remaining-microalgae biomass turned to be colorless. The solvent was removed by letting it evaporated in the open air and the lipid content was determined gravimetrically. The extraction was performed in quadruplicate. The resulting percentage of extracted lipids is the sum of three extractions performed in series.

\section{Fatty acid profiles of microalgae strains}

Fatty acids profile was determined by the capillary column gas chromatographic with mass spechtrophotometer (GCMS) method applied to the oil methyl esters [14]. The amount of total fatty acids of each microalgae strain was obtained by transesterification into the corresponding methyl esters (fatty acid methyl esters (FAME)), through saponification with $\mathrm{NaOH}$ in methanol, followed by methylation with BF3 catalyst. The FAME then were extracted with $n$-hexane.

\section{RESULTS AND DISCUSSION \\ Growth and lipid accumulation properties}

Microalgal strains, Chlorophytes, Cyanobacteria, Diatoms, and Euglenoid, have been found to contain proportionally high levels of lipids (over 30\%). Micro- 
Table 1. Growth kinetics, lipid content, and lipid productivity of 3 microalgae strains

\begin{tabular}{|c|c|c|c|c|}
\hline Isolates code & $\begin{array}{c}\text { Specific growth rate } \\
\left(\text { day }^{-1}\right)\end{array}$ & $\begin{array}{c}\text { Biomass productivity } \\
\left(m g \cdot L^{-1} \cdot \text { day }^{-1}\right)\end{array}$ & Lipid content (\%) & $\begin{array}{c}\text { Lipid productivity } \\
\quad\left(m g \cdot L^{-1} \cdot \text { day }^{-1}\right)\end{array}$ \\
\hline LBB13-2-AL045 & $0.437 \pm 0.010$ & $68.13 \pm 0.88$ & $49.08 \pm 0.25$ & $33.43 \pm 0.60$ \\
\hline LBB13-2-AL046 & $0.106 \pm 0.008$ & $46.88 \pm 2.65$ & $24.6 \pm 0.16$ & $11.53 \pm 0.58$ \\
\hline LBB13-2-AL048 & $0.123 \pm 0.007$ & $115.72 \pm 6.99$ & $34.81 \pm 0.45$ & $40.27 \pm 1.91$ \\
\hline
\end{tabular}

Table 2. Fatty acid profiles of 3 strains microalgae

\begin{tabular}{|c|c|c|c|}
\hline Fatty acids & LBB13-2-AL045 & LBB13-2-AL046 & LBB13-2-AL048 \\
\hline C14:0 & - & 0.64 & 0.84 \\
\hline C15:0 & - & - & 10.22 \\
\hline $\mathrm{C} 16: 0$ & 29.11 & 42.24 & 15.77 \\
\hline C16:1 & 0.84 & 1.04 & 1.48 \\
\hline $\mathrm{C} 16: 2(\mathrm{n}-6)$ & 4.45 & 2.74 & 2.04 \\
\hline C16:4(n-3) & 4.73 & 7.32 & 1.98 \\
\hline C18:0 & 1.90 & 2.35 & 8.34 \\
\hline C18:1(n-9) & 43.81 & - & 8.22 \\
\hline C18:1(n-7) & - & - & 1.01 \\
\hline $\mathrm{C} 18: 2(\mathrm{n}-3)$ & - & 24.88 & - \\
\hline $\mathrm{C} 18: 2(\mathrm{n}-6)$ & 7.18 & - & 23.74 \\
\hline $\mathrm{C} 18: 3(\mathrm{n}-3)$ & 4.79 & 9.16 & 24.74 \\
\hline $\mathrm{C} 18: 3(\mathrm{n}-6)$ & - & 3.13 & 1.10 \\
\hline C18:4(n-3) & 1.89 & 3.78 & - \\
\hline $\mathrm{C} 20: 1(\mathrm{n}-7)$ & - & 1.09 & - \\
\hline $\mathrm{C} 20: 3(\mathrm{n}-6)$ & 0.63 & - & - \\
\hline $\mathrm{C} 20: 4(\mathrm{n}-6)$ & 0.67 & - & - \\
\hline C22:0 & - & 1.03 & - \\
\hline $\mathrm{C} 22: 5(\mathrm{n}-3)$ & - & - & 0.52 \\
\hline $\mathrm{C} 24: 0$ & - & 0.60 & - \\
\hline SFA & 31.01 & 46.86 & 35.17 \\
\hline MUFA & 44.65 & 2.13 & 10.71 \\
\hline PUFA & 24.34 & 51.01 & 52.08 \\
\hline UFA & 68.99 & 53.14 & 62.79 \\
\hline
\end{tabular}

Table 3. Nutritive value of 3 microalgae strain isolates

\begin{tabular}{|c|c|c|c|}
\hline & LBB13-2-AL045 & LBB13-2-AL046 & LBB13-2-AL048 \\
\hline$\sum_{\mathrm{n}-3}$ & 11.41 & 45.14 & 27.24 \\
\hline$\sum_{\mathrm{n}-6}$ & 12.93 & 5.87 & 26.88 \\
\hline$(n-6) /(n-3)$ & 1.13 & 0.13 & 0.99 \\
\hline
\end{tabular}


algal strains with high oil or lipid content are of great interest in the search for a sustainable feedstock for the production of bio-oil. According to Griffith and Harrison [16], lipid productivity is a key criterion for choosing best strain of microalgae as a lipid producer. Lipid productivity could be calculated by biomass productivity and lipid content. An investigation on selected three strains of tropical microalgae showed that LBB13-2AL045 strain exhibited the fastest specific growth rate $\left(0.437 \pm 0.010 \mathrm{day}^{-1}\right)$, followed by LBB13-2-AL048 $\left(0.123 \pm 0.007 \mathrm{day}^{-1}\right)$ and LBB13-2-AL046 (0.106 \pm 0.008 day $^{-1}$ ) (Table 1). Among other two strains, LBB13-2-AL045 strain showed superior ability to grow and to multiply its biomass.

Biomass productivity was calculated by the dry biomass (in grams per liter per day), during the exponential growth phase [14]. The results showed that LBB13-2-AL048 strain had the highest biomass productivity at $115.72 \pm 6.99 \mathrm{mg} \cdot \mathrm{L}^{-1} \cdot \mathrm{day}^{-1}$, while LBB13-2AL045 with lower biomass productivity $(68.13 \pm 0.88$ mg. $\mathrm{L}^{-1} \cdot$ day $^{-1}$ ) and LBB13-2-AL046 with the lowest biomass productivity $\left(46.88 \pm 2.65 \mathrm{mg} \cdot \mathrm{L}^{-1} \cdot \mathrm{day}^{-1}\right)($ Table $1)$. The biomass productivity of the three strains was not consistent with the specific growth rate. Although LBB13-2-AL048 showed not highest specific growth rate, its cell density was constantly increased in 13-day cultivation (late logarithmic phase) which may result in its highest biomass productivity.

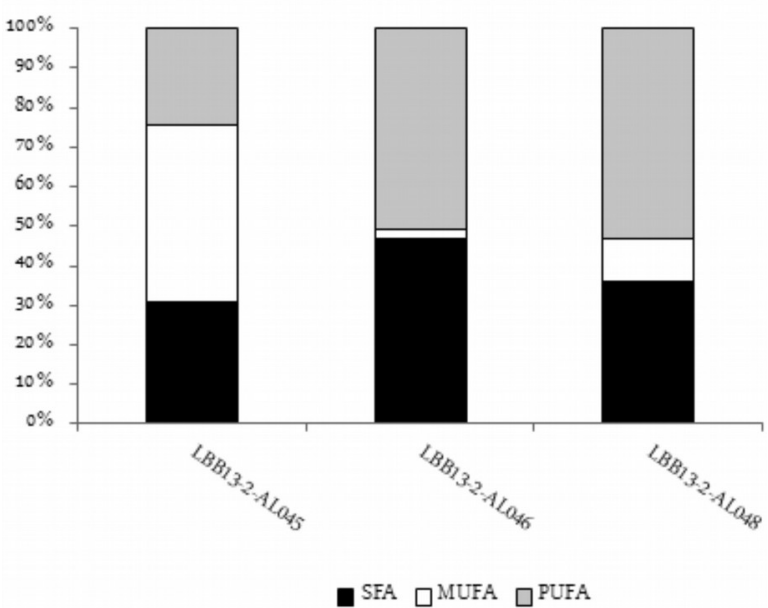

Figure 1. The percentage of fatty acids, monounsaturated fatty acids, polyunsaturated fatty acids and others in fatty acid compositions of 10 microalgal strains. SFA $=$ saturated fatty acids (14:0, 15:0, 16:0, 18:0, 22:0, 24:0); MUFA = monounsaturated fatty acids $(16: 1,18: 1,20: 1) ;$ PUFA $=$ polyunsaturated fatty acids $(16: 2,16: 4,18: 2,18: 3,18: 4,20: 3,20: 4,22: 5)$
Lipid productivity is not only calculated by the biomass productivity, but it is also calculated by the lipid content within microalgae cell. Lipid content of the 3 strains at the late stationary phase was analyzed. The results showed LBB13-2-AL045 attained the highest lipid content at $49.08 \pm 0.25 \%$, followed by LBB132-AL048 at $34.81 \pm 0.45 \%$ and LBB13-2-AL046 at 24.6 $\pm 0.16 \%$ (Table 1 ). Compared to the biomass productivity, we found the top biomass producers in the present study did not correspond to the top lipid accumulators. The phenomenon was consistent with the previous report $[17,18]$.

Combined with the biomass productivity, the lipid productivity of the 3 strains of freshwater microalgae was analyzed. The results showed that lipid productivity of LBB13-2-AL048 was the highest $(40.27 \pm 1.91$ mg. $\mathrm{L}^{-1} \cdot$ day $^{-1}$ ), followed by LBB13-2-AL045 (33.43 \pm 0.60 mg. $\mathrm{L}^{-1} \cdot$ day $^{-1}$ ), while LBB13-2-AL046 was the lowest $\left(11.53 \pm 0.58 \mathrm{mg} \cdot \mathrm{L}^{-1}\right.$.day $\left.{ }^{-1}\right)$ (Table 1$)$. Based on the lipid productivity, LBB13-2-AL048 strain could be considered as the best feedstock for microalgal lipid production in the 3 fast-growth freshwater microalgae isolated from Bengkulu province.

\section{Fatty acids profiles compositional properties}

Through analysis profiling of the fatty acids (FAs) composition data in Table 2, a useful comparison of the 3 algal lipids with respect to the saturated, monounsaturated, and polyunsaturated compounds is provided in Figure 1. The fatty acid compounds of 3 algal lipids were varied, especially for MUFA and PUFA. Fatty acids of monounsaturated fatty acids (MUFAs) ranged from $2.13 \%$ to $44.65 \%$ and Polyunsaturated fatty acids (PUFAs) from $24.34 \%$ to $52.08 \%$, while saturated fatty acids (SFAs) composition among 3 algal lipids is closely similar (Table 2.). In comparisons of the fatty acids composition between LBB13-2-AL045 and two other strains, MUFA (44.65\%) contents was relatively high and PUFA (24.34\%) contents was relatively low. In particular, C18:1 (43.81\%) of LBB13-2AL045 strain was higher than the other strains, while C18:2 (7.18\%) was lower than two other strains.

The fatty acids composition of two strains, LBB132-AL046 and LBB13-2-AL048, contain similar high percentage of PUFA. The high amount of PUFA of LBB13-2-AL046 and LBB13-2-AL048 strain made their algal oils was not suitable to be applied as biodiesel feedstock as it would likely result in low oxidative stability [19]. Instead, the algal oils produced from LBB13-2-AL046 and LBB13-2-AL048 strains could be potential to be used as omega- 3 and omega- 6 sources. 
Omega-3 and omega-6 contents of LBB13-2-AL046 strain, respectively, was $45.14 \%$ and $5.87 \%$; while of LBB13-2-AL048 strain, respectively, was $27.24 \%$ and $26.88 \%$. The high content of omega- 3 and omega-6 fatty acids indicated that the algal oils of two strains was potential to be applied in health application, especially cardiovascular health [20]. On the other hand, LBB13-2-AL045 strain contains relatively low of omega-3 (11.41\%) and omega-6 (12.93\%), but its fatty acid profile (SFA : MUFA : PUFA) has a balance ratio, close to $1: 1.3: 1$ as is recommended by American Heart Association (AHA) [20]. Therefore, according to Hayes [20], the algal oil of LBB13-2-AL045 strain was recommended in order to generate the best $\mathrm{LDL} / \mathrm{HDL}$ ratio.

On the other hand, all strains presented a highly SFA profile in which the percentage of palmitic acid (C16:0) is the highest compared to another SFA and it is ranged from $15.77 \%$ (LBB13-2-AL048) to 42.24 (LBB13-2-AL046) (Table 2). Many studies have suggested that SFA has negative effect for cardiovascular health since it could raise total cholesterol (TC), LDL (Low Density Lipoprotein) and HDL (High Density Lipoprotein) [20], and, therefore, it is recommended to reduce its content in the diet [21].

\section{Possible use of algal oils in health application}

Microalgae are considered as an alternative source of essential fatty acids, particularly omega-3, in order to replace fish oil [22]. The use of microalgae as essential fatty acids source provides many benefits, not only for human health, but also for the effectiveness and efficiency production process, since they are exceedingly rich in oil and can grow extremely rapid [23]. Three strains analyzed in our study are fast-growing microalgae and, therefore, could offer advantages if they are used as oil producer. In addition, their lipid content was also high, resulted in high lipid productivity (Table 1). In view of Table 3 , three microalgae strains showed that its omega-3 fatty acids contents were quite high, while omega- 6 fatty acids content was in moderate level. Omega-3 and omega-6 fatty acids may prevent coronary heart disease [24] and stroke [25] as they have previously been shown to reduce blood cholesterol levels [26] and improve hypertension [27,28].

However, the ratio of omega- 6 to omega- 3 essential fatty acids is important for human's health due to the excessive amounts of omega- 6 fatty acids and a very high omega-6/omega-3 ratio promote the pathogenesis of many diseases, including cardiovascular disease, cancer, and inflammatory and autoimmune diseases [29].
On the other hand, the increasing levels of omega- 3 fatty acids (a low omega-6/omega-3 ratio) could be beneficial due to their anti-inflammatory properties and plaque-stabilizing effects [29,30]. In this study, oil from LBB13-2-AL048 strain possesses balance ratio of omega-6/omega-3 (close to 1) which is good for human consumption in order to maintain their health state, whereas oil from LBB13-2-AL046 strain that possesses low omega-6/omega-3 (0.13, Table 3) may be beneficial to suppress inflammatory effect of omega- 6 . In industrial countries, the amount of ingested of omega- 6 has been reported to increase dramatically by $240 \%$ over the last 40 years [31]. Therefore, oil from LBB13-2-AL046 could be utilized as edible oil to increase the amount of ingested of omega- 3 due to its high content of omega-3 fatty acids (45.14\%). Moreover, as observed by Blanchard et al. [32], increasing dietary omega-3 (Alpha-Linolenic Acid or ALA) and reducing omega-6 (Linoleic Acid or LA) intake were both beneficial in increasing omega-3 Long ChainPolyunsaturated Fatty Acids (LC-PUFA) bioavailability in tissues, including led to a higher proportion of DHA in brain and heart.

Research conducted by Weisweiler et al. [33] suggested the importance of dietary fatty acid balance on the lipoprotein profile. On the other hand, AHA and National Cholesterol Education Program (NCEP) recommend the approximately equal balance of SFA : MUFA : PUFA ( $1: 1.3: 1)$ as a basic consideration at any fat intake for maintaining ideal LDL/HDL ratio (low LDL and high HDL for any given Total Cholesterol value) [20]. In this study, it is known that oil from LBB13-2- AL045 possesses close-to-balance fatty acids profile of SFA : MUFA : PUFA $(1: 1.4: 0.8)$ as is recommended by AHA and NCEP. The balance ratio of fatty acids profile is critical at any level of fat intake if one wishes to avoid adversely affecting the lipoprotein profile [20]. The high content of MUFA in LBB132-AL045's oil is also beneficial for health, particularly for heart health, due to its lowering effect of total- , LDL-cholesterol, and plasma triglyceride levels and also increasing effect of HDL cholesterol levels. In addition, a diet high in MUFA improves glycemic control in individuals with noninsulin-dependent diabetes mellitus [34]. Based on its fatty acids content, oil from LBB13-2-AL045 has similar profile with palm-oil's fatty acids, but with higher of PUFA and lower of SFA content. Therefore, oil from LBB13-2-AL045 could offer more benefits over palm oil, especially for human's health. Thus, LBB13-2-AL045's oil could be used as an alternative source for cooking oil feedstock in order to 
replace palm oil.

\section{CONCLUSION}

The characterization of three microalgae strains isolated from tropical lake of Bengkulu, Sumatra, Indonesia has depicted that the microalgae could be used as source for the good lipid and fatty acids of PUFA and MUFA. The two strains of LBB13-2-AL046 and LBB132-AL048 were possesses valuable fatty acids of omega-3 and omega-6, whereas LBB13-2-AL045 synthesized high MUFA content. The fatty acids profile analysis of the three strains showed that their oil could possibly be used to improve human's health, particularly cardiovascular health.

\section{ACKNOWLEDGMENT}

The authors gratefully acknowledged to technician in Bioenergy and Bioprocess Laboratory, Research Center for Biotechnology, LIPI (Ms. Fitriyani) for her good assistance.

\section{REFERENCES}

1. Herrera-Valencia VA, Contreras-Pool PY, Lopez-Adrian SJ et al (2011) The green microalgae Chlorella saccharophila as a suitable source of oil for biodiesel production. Current Microbiology. 63: 151-157.

2. Spolaore P, Joannis-Cassan C, Duran E et al (2006) Commercial application of microalgae. Journal of Bioscience and Bioengineering. 101: 87-96.

3. Milledge JJ (2011) Commercial application of microalgae other than as biofuels: a brief review. Rev Environ SciBiotechnol. 10: 31-41.

4. Bigogno C, Khozin-Goldber I, Boussiba S et al (2002) Lipid and fatty acid composition of the green oleaginous alga Parietochlorisincisa, the richest plant source of arachidonic acid. Phytochemistry. 60: 497-503.

5. Adarme-Vega TC, Lim DKY, Timmins M et al (2012) Microalgalbiofactories: a promising approach towards sustainable omega-3 fatty acid production. Microbial cell factories. 11: 96-105.

6. Chen G, Jiang Y, Chen F (2007) Fatty acid and lipid class composition of eicosapentaenoic acid-producing microalga, Nitzchialaevis. Food chemistry. 104: 1580-1585.

7. Cohen Z (1999) Production of polyunsaturated fatty acids by the microalga Porphyridium cruetum. In: Cohen Z, ed.Chemicals from microalgae. London, Taylor and Francis. 124.

8. Raposo MFdJ, de Morais RMSC, de Morais AMMB (2013) Health application of bioactive compounds from marine microalgae. Life Sciences. 93: 479-486.

9. Sjitsma L, de Swaaf ME (2004) Biotechnological production and applications of the omega-3 polyunsaturated fatty acid docosahexaenoic acid. Appl Microbiol Biotechnol. 64:146153.

10. Tonon T, Harvey D, Larson TR et al (2002) Long chain polyunsaturated fatty acid production and partitioning to triacylglycerols in four microalgae. Phytochemistry. 61:1524.

11. Pulz O, Gross W (2004) Valuable products from biotechnology of microalgae. Appl Microbiol Biotechnol. 65:635648.

12. Gerber LR, Karimi R, Fitzgerald TP (2012) Sustaining seafood for public health. Front Ecol Environ. 10 (9): 487493.

13. Brett MT, Muller-Navarra DC (1997) The role of highly unsaturated fatty acids in aquatic foodweb process. Freshwater Biology. 38:483-499.

14. Nascimento IA, Marques SSI, Cabanelas ITD et al (2013) Screening microalgae strains for biodiesel production: Lipid productivity and estimation of fuel quality based on fatty acids profiles as selective criteria. Bioenerg Res. 6:1-13.

15. Ryckebosch E, Muylaert K, Foubert I (2012) Optimization of an analytical procedure for extraction of lipids from microalgae. J Am Oil Chem Soc. 89:189-198.

16. Griffiths MJ, Harrison STL( 2009) Lipid productivity as a key characteristics for choosing algal species for biodiesel production. J. Appl. Phycol. 21:493-507.

17. Ma Y, Wang Z, Yu C et al (2014) Evaluation of the potential of 9 Nannochloropsis strains for biodiesel production. Biores Technol. 167:503-509.

18. Song M, Pei H, Hu W et al (2015) Evaluation of the potential of 10 microalgal strains for biodiesel production. Biores. Technol. 141:245-251.

19. Knothe G (2009) Improving biodiesel properties by modifying fatty esters composition. Energy Environ Sci. 2:759-766.

20. Hayes KC (2002) Dietary fat and heart health: in search of the ideal fat. Asia Pacific J Clin Nutr. 11: 394-400.

21. Micha R, Mozaffarian D (2010) Saturated fat and cardiometabolic risk factors, coronary heart disease, stroke, and diabetes: a fresh look at the evidence. Lipids. 45: 893-905.

22. Solana M, Rizza CS, Bertucco A (2014) Exploiting microalgae as a source of essential fatty acids by supercritical fluid extraction of lipids: comparison between Scenedesmus obliquus, Chlorella protothecoides and Nannochloropsis salina. The Journal of Supercritical Fluids. 92:311-318.

23. Chisti Y (2007) Biodiesel from microalgae. Biotechnology Advances. 25: 294-306.

24. Mozaffarian D, Appel LJ, van Horn L (2011) Components of a cardioprotective diet: New insights. Circulation. 123: 2870-2891.

25. Navab M, Ananthramaiah GM, Reddy ST et al (2004) The oxidation hypothesis of atherogenesis: The role of oxidized 
phospholipids and HDL. Journal of Lipid Research. 45 (6): 993-1007.

26. Apostolopoulou M, Michalakis K, Miras A et al (2012) Nutrition in the primary and secondary prevention of stroke. Maturitas. 72: 29-34.

27. Bao DQ, Mori TA, Burke V et al (1998) Effects of dietary fish and weight reduction on ambulatory blood pressure in overweight hypertensives. Hypertension. 32: 710-717.

28. Hu FB, Bronner L, Willet WC et al (2002) Fish and omega3 fatty acid intake and risk of coronary heart disease in women. J Am Med Assoc. 287: 1815-1821.

29. Simopoulos AP (2002) The importance of the ratio of omega-6/omega-3 essential fatty acids. Biomedicine Pharmacotheraphy. 56: 365-379.

30. Raposo MFJ, de Morais AMMB (2015) Microalgae for the prevention of cardiovascular disease and stroke. Life Sci- ences. 125: 32-41.

31. Dunbar BS, Bosire RV, Deckelbaum RJ (2014) Omega 3 and omega 6 fatty acids in human and animal health: An African perspective. Molecular and Cellular Endocrinology. 398: 69-77.

32. Blanchard H, Pedrono F, Boulier-Monthean N et al (2013) Comparative effects of well-balanced diets enriched in alpha-linolenic or linoleic acids on LC-PUFA metabolism in rat tissues. Prostaglandins, Leukotrienes and Essential Fatty Acids. 88: 383-389.

33. Weisweiler P, Janetschek P, Schwandt P (1985) Influence of polyunsaturated fats and fat restriction on serum lipoproteins in humans. Metabolism. 34: 83-87.

34. Kris-Etherton P (1999) Monounsaturated fatty acids and risk of cardiovascular disease. Circulation. 100: 1253-1258. 